
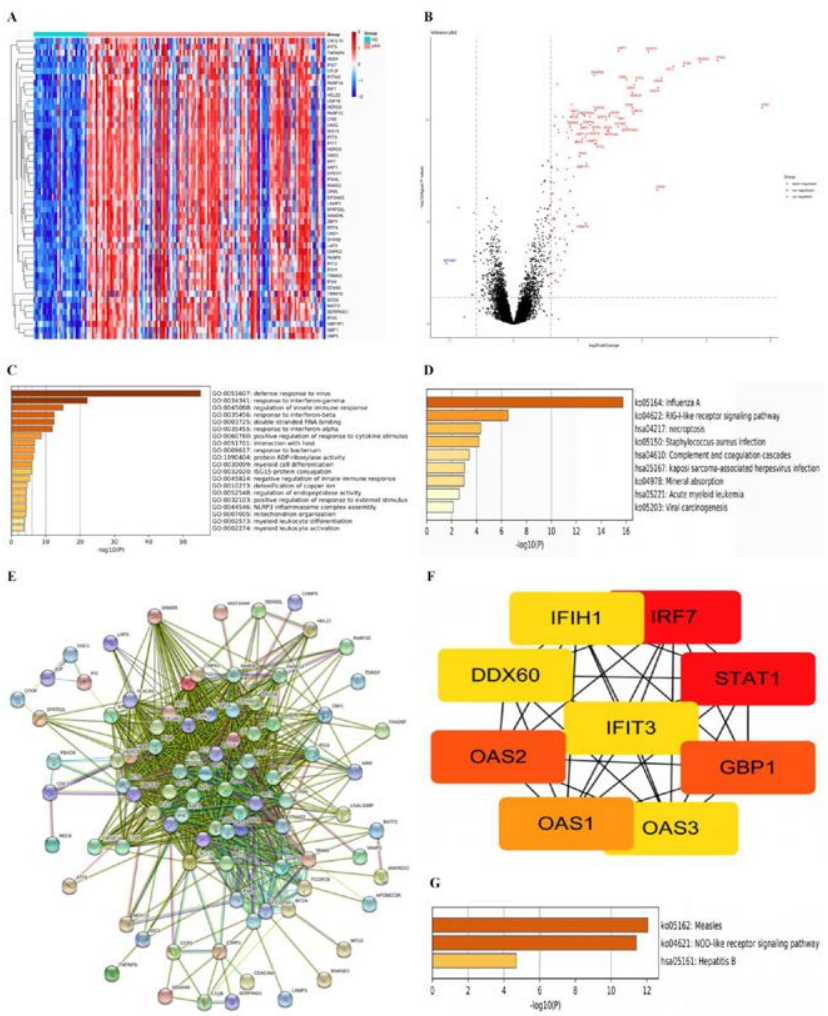

Figure I: (A)Heat map of the top 50 up-regulated differentially espressed genes: Each small square represents a gene: the color indicates

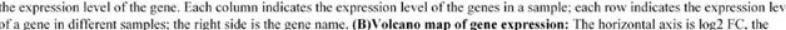
vertical axis is log 10 adj.P.V. Val. Each dot represents a genc, bluc indicates low expression genes, red indicates high. (C,D)V isualization GO enrichment analysis and KEGG pathway enrichment analysis. (E.F.G)PPI network and hub gene identification: Top 9 lub genes in the PPI network were screened by Cytoscape plugin cytoHubba based on their degree value from red (high degree value) to yellow (low degree

Acknowledgements: This project was supported by National Science Foundation of China (82001740), Open Fund from the Key Laboratory of Cellular Physiology (Shanxi Medical University) (KLCP2019) and Innovation Plan for Postgraduate Education in Shanxi Province (2020BY078).

Disclosure of Interests: None declared

DOI: 10.1136/annrheumdis-2021-eular.2021

\section{POS0743 GENE EXPRESSION MICROARRAY IN LUPUS NEPHRITIS BY BIOINFORMATIC ANALYSIS}

C. Wang ${ }^{1,2,3}$, S. X. Zhang ${ }^{1,2,3}$, S. Song ${ }^{1,2,3}$, J. Qiao ${ }^{1,2,3}$, R. Zhao ${ }^{1,2,3}$, M. J. Chang ${ }^{1,2,3}$, Y. Zhang ${ }^{1,2,3}$, G. Y. Liu', P. F. He ${ }^{4}$, X. Li ${ }^{1,2,3} .{ }^{1}$ The Second Hospital of Shanxi Medical University, Department of Rheumatology, Taiyuan, China; ${ }^{2}$ Shanxi Li Xiaofeng Medical Groups, Department of Rheumatology, Taiyuan, China; ${ }^{3}$ Ministry of Education, Key Laboratory of Cellular Physiology at Shanxi Medical University, Taiyuan, China; ${ }^{4}$ Shanxi Medical University, Medical Data Sciences, Taiyuan, China

Background: Nephritis is one of the predominant causes of morbidity and mortality in patients with lupus ${ }^{12}$. The lack of understanding regarding the molecular mechanisms of lupus nephritis(LN) hinders the development of specific targeted therapy for this progressive disease ${ }^{3}$.

Objectives: In this study, we use bioinformatics method to analyze the genes involved in regulating the potential pathogenesis of LN.

Methods: The expression profile of LN(GSE104948 and GSE32591) was obtained from the GEO database.GSE104948 was a memory chip, which included 32 LN glomerular biopsy tissues and 3 glomerular tissues from living donors.GSE32591 dataset included 32 LN glomerular biopsy tissues and 15 glomerular tissues from living donors. The Oligo package was used to process the data to obtain the expression matrix files of all the related genes.P $<0.05$ and $|\log 2(F C)|>2$ were setted as cut-off criteria for the DEGs.Ggplot2, heatmap packages were used to DEGs visualization. Metascape online tool was used to annotating DEGs for Gene Ontology (GO) and Kyoto Encyclopedia of Genes and Genomes (KEGG) pathway enrichment analysis performed.We used STRING online database to construct protein-protein interaction (PPI) network. Hub genes were identified by Cytoscape.

Results: In differential expression analysis,357 DEGs were identified,including 248 up-regulated genes and 109 down-regulated genes (Figure 1A,B). GO enrichment showed that these DEGs were primarily enriched in biological pathways, cell localization and molecular function and revealed that LN-related genes mainly involved in immune response.KEGG pathway annotation enrichment analysis revealed these DEGs were closely associated with Staphylococcus aureus infection,Complement and coagulation cascades (Figure 1D) Fourteen hub genes(IFT3,IRF7,OAS3,GBP2,RSAD2,MX1,IFIT2,IFI6,MX2,IS F15,IFIT1,QAS2,OASL,OAS1) were identified from PPI network (Figure 1C,E).

Conclusion: Illuminating the molecular mechanisms of LN was help for deep understanding of LN.

REFERENCES:

[1] Song J, Zhao L, LiY. Comprehensive bioinformatics analysis of mRNA expression profiles and identification of a miRNA-mRNA network associated with lupus nephritis. Lupus 2020;29(8):854-61. doi: 10.1177/0961203320925155 [published Online First: 2020/05/22].

[2] Yao F, Sun L, Fang W, et al. HsamiR3715p inhibits human mesangial cell proliferation and promotes apoptosis in lupus nephritis by directly targeting hypoxiainducible factor 1alpha. Mol Med Rep 2016;14(6):5693-98. doi: 10.3892/mmr.2016.5939 [published Online First: 2016/11/24].

[3] Dall'Era M. Treatment of lupus nephritis: current paradigms and emerging strategies. Curr Opin Rheumatol 2017;29(3):241-47. doi: 10.1097/ BOR.0000000000000381 [published Online First: 2017/02/17].
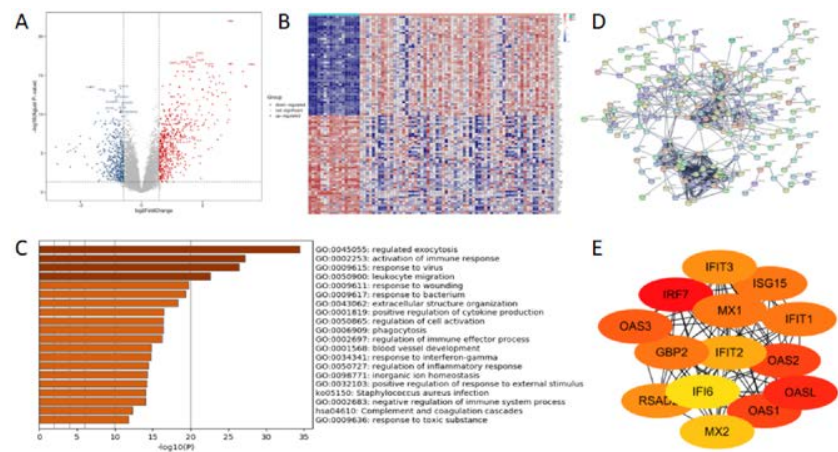

Figurel:(A)Volcano plots of DEGs between LN and healthy controls.Each dot represents a gene, where red represent up-regulated, blue represent down-regulated genes, and black represent genes with no significant represent up-regulated, blue represent down-regulated genes, and black represent genes with no significant
difference.(B)Heatmap of the top 50 up-regulated and 50 down-regulated DEGs.Red represent up-regulated, blue represent down-regulated genes. Each row represents the expression level of a gene in a sample, sample grouping information is on the right. Each column represents the expression level of a gene in different samples.(C)Heatmap of enriched terms across input gene lists, colored by p-values.(D,E)PPI network and hub gene identification.Top 14 hub genes in the PPI network were screened by Cytoscape plugin cytoHubba based on their degree value from red (high degree value) to yellow (low degree value).

Acknowledgements: This project was supported by National Science Foundation of China (82001740), Open Fund from the Key Laboratory of Cellular Physiology (Shanxi Medical University) (KLCP2019) and Innovation Plan for Postgraduate Education in Shanxi Province (2020BY078).

Disclosure of Interests: None declared

DOI: 10.1136/annrheumdis-2021-eular.2062

\section{POS0744 A NEGATIVE INTERFERON BIOMARKER CD169 I SIGLEC-1 RULES OUT SYSTEMIC LUPUS ERYTHEMATOSUS}

L. Zorn-Pauly ${ }^{1}$, A. S. L. Von Stuckrad ${ }^{2}$, J. Klotsche ${ }^{3}$, T. Rose ${ }^{1}$, T. Kallinich ${ }^{2}$ F. Hiepe ${ }^{1}$, P. Enghard ${ }^{4}$, L. Ostendorf ${ }^{4}$, T. Dörner ${ }^{1}$, C. Meisel ${ }^{5}$, U. Schneider ${ }^{1}$, N. Unterwalder ${ }^{5}$, G. R. Burmester ${ }^{1}$, T. Alexander ${ }^{1}$, R. Biesen ${ }^{1} .{ }^{1}$ Charité Universitätsmedizin Berlin, Rheumatology and Clinical Immunology, Berlin, Germany; ${ }^{2}$ Charité - Universitätsmedizin Berlin, Pediatric Pneumology and Immunology, Berlin, Germany; ${ }^{3}$ German Rheumatism Research Centre, Epidemiologic Unit, Berlin, Germany; ${ }^{4}$ Charité - Universitätsmedizin Berlin, Nephrology and Intensive Care Medicine, Berlin, Germany; ${ }^{5}$ Institute for Medical Immunology, Labor Berlin - Charité Vivantes, Immunology, Berlin, Germany

Background: While there have been advances in the therapy of systemic lupus erythematosus (SLE) in recent years, there have been no major new findings in SLE biomarkers [1, 2]. Type I interferon (IFN) plays a pivotal role in the pathogenesis of SLE [3]. In 2008, we first described CD169 / SIGLEC-1 (sialic acid-binding immunoglobulin-like lectin-1), an interferon-induced adhesion molecule on monocytes in SLE patients [4]. For over five years SIGLEC-1 has been routinely assessed in our clinic.

Objectives: To evaluate and compare the diagnostic utility of the type I IFN induced SIGLEC-1 with established biomarkers in the initial diagnosis of the disease.

Methods: We analyzed retrospectively 232 patients who were on suspicion of SLE at Charité University Hospital Berlin between October 2015 and September 2020. Patients underwent full clinical characterization, and biomarkers were 